\title{
Başkalaşan Kanat Ucu Tasarımı ve Avantajları
}

\author{
Hüseyin Şahin ${ }^{1 *}$, Tuğrul Oktay ${ }^{2}$ \\ ${ }^{1}$ Ankara Yıldırım Beyazıt Üniversitesi, Meslek Yüksekokulu, Uçak Teknolojisi Bölümü, Ankara, Türkiye (ORCID: 0000-0003-0464-2644) \\ ${ }^{2}$ Erciyes Üniversitesi, Havacılık ve Uzay Bilimleri Fakültesi, Uçak Mühendisliği Bölümü, Kayseri, Türkiye (ORCID: 0000-0003-4860-2230)
}

(Ilk Geliş Tarihi 19 Ekim 2019 ve Kabul Tarihi 2 Kasım 2019)

(DOI: 10.31590/ejosat.634822)

\begin{abstract}
ATIF/REFERENCE: Şahin, H., \& Oktay, T. (2019). Başkalaşan Kanat Ucu Tasarımı ve Avantajları. Avrupa Bilim ve Teknoloji Dergisi, (17), 606-610.

Öz

Kanat ucunda meydana gelen vorteks drag(sürüklemesinin) azaltılması için hava aracı üreticileri winglet veya sharklet adını verdikleri kanat ucunın kıvrılması formunda yapılar tasarlamışlardır. Bu makalede ise yine sabit kanatlı hava araçları için aerodinamik performans artırmak için kıvrık kanat ucu tasarımının geliştirilip hareketli kanat ucu tasarımı hakkında çalışılmıştır. Sabit kanatlı hava aracı üreten büyük firmalar değişik tiplerde ve formlarda kıvrık kanat yapısı denemiş ve kullanmışlardır. Tasarlanan ve kullanılan değişik tiplerdeki kıvrık kanat uçlarının farklı aerodinamik karakteristikleri mevcuttur. Airbus, Boeing gibi büyük uçak üretici firmalar yakıt tasarrufu gibi avantajlarından dolayı kıvrık kanat ucunu tasarlamışlardır. Kıvrık kanat ucu tasarımı uçuşun büyük bir kısımı olan düz uçuş safhası için iyi bir itki-sürtünme kuvveti oranı sağlayarak büyük bir verim sağlamasına rağmen uçuşun bütün safhaları için uygun yakıt performans değeri sağlamayacaktır. Düşük hızlarda vorteks sürtünme az olduğundan dolayı özellikle kalkış ve iniş gibi düşük hızda yüksek kaldırma kuvveti istenen durumlar için kıvrık kanat ucu tasarımının avantajı çok görülmemektedir. Bu nedenle düşük hızlarda kullanılmak üzere kıvrık kanat ucunun formunu kanat ile benzer açıya çekip kaldırma kuvveti artışı yapılabilir. Kanat ucu şeklinin değişimine izin veren başkalaşan kanat ucu tasarımı, kanat ucundaki bir menteşe yardımıyla hareket etmesine olanak vermektedir. Bu sayede düşük hızlarda daha fazla kaldırma kuvveti oluşturacak şekilde, düz uçuş safhasında ise daha az sürtünme kuvveti oluşturacak şekilde tasarlanmıştır. Kanat ucuna yapılan bu ufak mekanik değişiklik uçuş performansına aerodinamik olarak olumlu yönde etkisinin yanı sıra hava aracının yakıt tasarrufu, menzil artışı, daha az karbon salınımı gibi birçok avantajı bulunmaktadır. Bu çalışmada başkalaşan kanat ucu tasarımının literatür taraması yapılmış ve başkalaşabilen kıvrık kanat ucu tasarımının avantajlarından bahsedilmiştir.
\end{abstract}

Anahtar Kelimeler: İnsansız Hava Aracı(İHA), Sabit Kanat, Başkalaşan kanat ucu.

\section{Morphinglet Wing Tip Design and Advantages}

\begin{abstract}
In order to reduce the vortex drag at the wing tip, aircraft manufacturers have designed structures in the form of curving wing tip, which they call the winglet or sharklet. In this article, the curved wing tip design has been developed and the moving wing tip design has been studied to improve aerodynamic performance for fixed wing aircraft. Producing fixed wing aircraft have tried and used curved wing structure in different types and forms. Different types of curved wing tip designed have different aerodynamic characteristics. International aircraft manufacturers such as Airbus and Boeing have designed the curved wing tip because of its fuel saving advantages. The curved wing tip design provides a good thrust-to-drag ratio for a large part of the flight, but it doesn't provide a suitable fuel performance value for all phase of flight. Because of low vortex drag at low speeds, the advantage of the curved blade tip design is not particularly noticeable, especially in situations where high lifts are required at low speeds such as take-off and landing. For this reason, the lifting force can be increased by the form of wing tip to a similar angle with the wing for use at low speeds. It is allowed to change the angle of the curved wing tip by use of a hinge and servo actuator system placed on the end of wing. Thus, it is designed to generate more lifting force at low speeds and less drag in the cruise flight phase. This small mechanical modification to the wing tip provides aerodynamically positive effects on flight performance, as well as many advantages such as
\end{abstract}

\footnotetext{
${ }^{*}$ Sorumlu Yazar: Ankara Yıldırım Beyazıt Üniversitesi, Meslek Yüksekokulu, Uçak Teknolojisi Bölümü, Ankara, Türkiye, ORCID: 0000-00030464-2644, husahin@ybu.edu.tr
} 
aircraft fuel savings, range increase and reduced carbon emissions etc.. In this study, literature review of morphinglet wing tip design has been made and advantages of morphinglet wing tip design.

Keywords: Unmanned Aerial Vehicle, Fixed Wing, Morphinglet.

\section{Giriş}

İnsanlar uçmayı kuşları gözlemleyerek öğrendikleri gibi uçuş performansını artırmayı da kuşları inceleyerek yapmaya devam etmektedir. Kuşlar uçuşlarını daha verimli yapabilmeleri için kanat şekillerini ve boyutlarını değiştirmektedirler. Hava aracı tasarımcıları kanat tasarlarken bu kuşları inceleyerek yapılmıştır. Kısa ve eliptik kanat şekline sahip şahin, sülün, ağaçkakan, güvercin gibi kuşlar hızlı kalkış ve kolay manevra kabiliyetlerine sahiptir, tıpkı STOL hava araçlarında olduğu gibi. Boşluksuz (slot) uzun kanata sahip kartal gibi kuşlar hızlı ve düz uçuş kabiliyetleri kuvvetlidir. Uzun dar kanatlı martı, kırlangıç gibi hayvanlar ise yüksek hızlarda süzülmeye izin verir. Kuşların kanat yapıları ve uçuş karakteristikleri incelenerek ve geliştirilerek aerodinamik verimliliğe sahip kanatlar tasarlamamıza yardımcı olacaktır. Hava araçları havada tutunabilmesi için kanadın altından geçen hava ile kanadın üstünden geçen havanın hızları ve basınçları farklı olması gerekir. Bu basınç farkı nedeni ile kanat ucunda vorteks sürüklemesi oluşur. Vortekslerin azaltılması için hava aracı üreticileri winglet/sharklet adını verdikleri kıvrık kanat ucu kullanmaktadır. Hava aracı üreticileri değişik formlarda kıvrık kanat yapısı üretmişlerdir. Özellikle sabit kanatlı hava araçlarının uçuş karakteristiklerini olumlu yönde etkileten Hava aracı üreticileri değişik formlarda kıvrık kanat yapısı üretmişlerdir. Bu uçuş safhaları genellikle kazaların en çok karşılaşıldığı uçuşun önemli kısımları olduğundan gerektiği zaman daha fazla kaldırma kuvveti oluşturulabilmesi için kanat ucunun şeklinin ve biçiminin değiştirilmesi verimliliği artıracaktır. Kanat ucu şeklinin değişimine izin veren başkalaşan kanat ucu, kanat ucunun katlanmasını sağlamaktadır. Geleneksel tasarımlardaki kanat yapılarında hava araçları statik kanat yapısı kullanır. Hava araçları kalkış ve iniş safhaları gibi düşük hızdaki performans gereksinimleri için yüksek kaldırma katsayısı ve düz uçuş için düşük sürtünme kuvveti gereksinimi vardır. Başkalaşabilen kıvrık kanat ucunda düşük hızlarda daha fazla kaldırma kuvveti oluşturacak şekilde, düz uçuş şartlarında ise daha az sürtünme kuvveti oluşturacak şekilde tasarlanmıştır. Bu çalışmada başkalaşabilen kanat ucuna sahip hava aracının hesaplamalı akışkanlar dinamiği ile analizi yapılmıştır.

\section{2. İnsansiz Hava Araçları Hakkında Genel Bilgiler}

Popüler bilim dallarından biri olan insansız hava araçlarının birçok avantajı bulunmaktadır. Bu avantajlardan en önemlileri maliyet, operatörünü taşımaması ve isteğe göre tasarlanabilir olmasıdır. Askeri ve sivil amaçlı birçok işlerde kullanıma uygun olarak tasarlanmıştır. Kullanıldıkları yerlere göre yakıtları elektrikli, sıvı yakıtlı veya hibrit olabilmektedir. İha sınıflandırmalarında birçok kriter bulunmaktadır. Bunlardan en yaygın olarak kullanılanı uçuş menzili, havada kalış süresi ve irtifadır. Uçuş menzillerine göre pointer gibi $100 \mathrm{~km}$ altı olan hava araçları kısa menzilli, silver fox gibi $100 \mathrm{~km}-400 \mathrm{~km}$ menzile sahip hava araçları orta menzilli, predator gibi $1500 \mathrm{~km}$ üzeri menzile sahip hava araçları ise uzun menzilli hava araçları kategorisi altında değerlendirilmektedir. Çıkabilecekleri maksimum irtifa açısından $1 \mathrm{~km}$ irtifaya yükselebilen hava araçları alçak irtifa, $1 \mathrm{~km}-10 \mathrm{~km}$ irtifaya yükselebilen hava araçları orta irtifa, 10kmden daha yüksek irtifaya çıkabilen hava araçları yüksek irtifa kategorisindedir.

\section{Literatür Taramasi}

Sabit kanatlı hava araçlarının performans optimizasyonu yapılarak daha verimli hava aracı üretmek mümkündür. $\mathrm{Bu}$ optimizasyon araştırmaları kullanılan malzemenin değişimi ile mümkün olabileceği gibi tasarımın değişimi ile de mümkün olmaktadır. Bu çalışmalar yapılarak aerodinamik performans artar ve yakıt tüketimi düşmesi gibi avantajlar sağlanmış olur.

İnsansız hava araçları askeri ve sivil amaçlar ile birçok görevlerde kullanılmaktadır. İnsansız hava aracı kullanımının avantajlarından bazıları işe uygun şekilde imal edilmeye uygun olması ve maliyetinin ucuz olması. Uçuşun daha verimli olması için birçok çalışmalar yapılmıştır.

Hava araçları birden fazla uçuş koşullarına elverişli olması gerek. Bir tasarım, belirli bir durumda en iyi performansı elde etmek için optimize edilebilse de, tüm uçuş koşullarında hiçbir tasarım ideal olamaz. Bu nedenle değişik uçuş koşullarına adapte olabilen sistemler üzerinde çalışmalar yapılmaktadır. Bu çalışmalardan birisi de başkalaşan kanat ucu tasarımıdır. Sabit kanatlı hava araçlarında kanat ucu tasarımı kullanımı sürükleme kuvvetini azaltmada önemli bir etkisi bulunmaktadır.

Başkalaşan kanat uçlarının uçak performansını arttırmak ve çalışma sürelerini uzatmak için kullanımı, yukarıda sunulan statik kanatçıkların içsel sınırlamalarının üstesinden gelmektedir. Bu yazıda sunulan morphing kanat ucu sistemi, İHA'nın iyileştirilmesi için bu firsatı ele almaktadır.

$\mathrm{Bu}$ çalışma, sabit kanatlı İHA'nın uçuşu görevi sırasında kullanılmak üzere kanat ucu şeklinin değiştirilmesi üzerine bir mekanizmanın tasarımını ve geliştirilmesini amaçlar. Böyle bir sistemin avantajları aşağıda açıklanmıştır ve bu proje içindeki sonuçlar, sistemin ve yapıların uçuş görevi boyunca karşılaşılan beklenen kuvvetlerle başa çıkma kabiliyetini göstermeye çalışmıştır.

Modern sabit kanatlı hava araçlarının kanatlarının uçlarında, kuşlardan ilham alınarak yapılmış winglet adı verilen yapılar mevcuttur. Bu winglet yapısı, kanat uçlarındaki tüylerinin neredeyse dikey bir açıda kıvrılabilen kuşların kanatlarından ilham almaktadir. 
Kanatların aerodinamik yapısı gereği, üst ve alt yüzey arasındaki basınç farkı, kanat uçlarında girdaplar oluşturmasına neden olur, bu girdaplar uçağın sürüklenmesine neden olur. "Tipik bir uçağının sürüklenme dağılımı, kaldırma kuvvetinin neden olduğu sürükleme düz uçuş koşullarında toplam sürüklemenin \% 40'ını ve kalkış sırasındaki toplam sürüklemenin \%80-90'ını oluşturabildiğini bilinmektedir”. [1]

\section{Şekil 1. Değişik şekil ve tiplerde kıvrık kanat ucu tasarımı[2]}

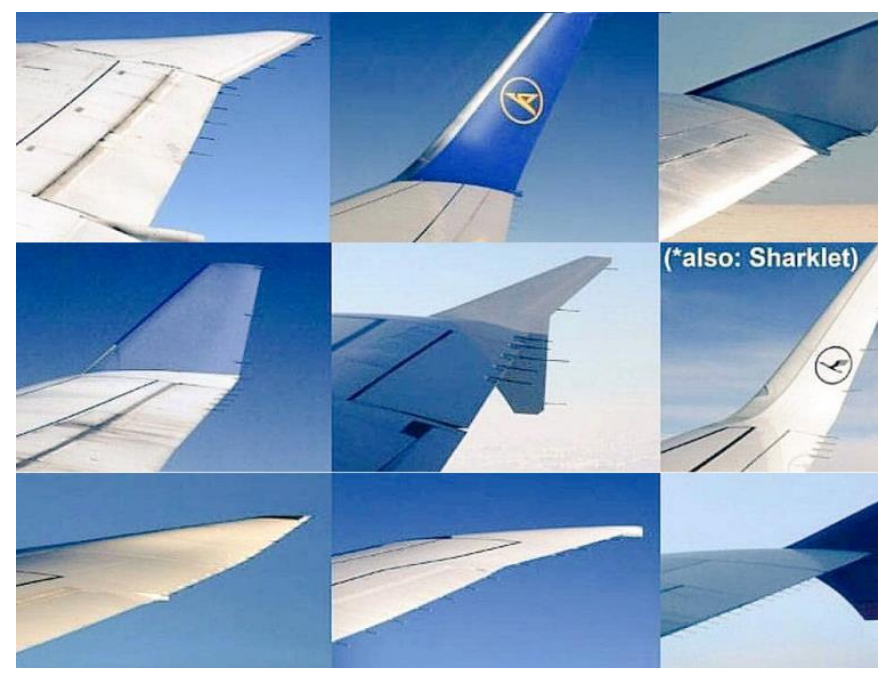

Başkalaşan kanat ucu tasarımı askeri ve sivil hava araçları için geliştirilmekte olan aerodinamik şekil değiştiren sistemdir. Başkalaşan kanat ucu tasarımı yakıt tasarrufu, verim ve manevra kabiliyeti avantajları sağlamaktadır. Petrol fiyatlarının artması ile birlikte ekonomik olarak rahatlamak isteyen kuruluşlar hava araçlarının daha verimli olmasını istemeleri sonucu ortaya çıkan çalışmalar neticesinde şekil değiştiren yapılar ortaya çıkmıştır. Yakıt tasarrufu sağlayan sistemler aynı zamanda çevresel olarak düşük karbon emisyonu sağlamaktadır.

\section{Şekil 2. Başkalaşan kanat ucu tasarımının çalışması [3]}

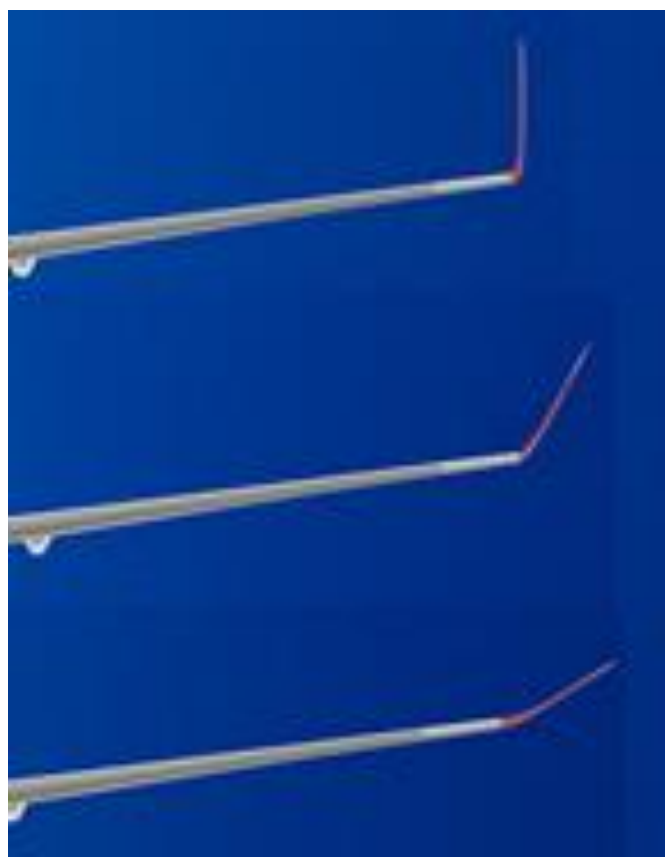

$\mathrm{Bu}$ çalışma sabit kanatlı hava araçlarının yakıt verimliliğini artırmak amacıyla kuş benzeri kanat ucu yapısının eğim açısını değiştirmeyi amaçlamaktadır. Böylelikle uçuşun her safhasında uçuş verimliliğini optimize etmektir.

\section{Sonuç}

Kıvrık kanat ucu tasarımı kullanımı İHAlarda sürüklemeyi önemli ölçüde azalttığı açıkça bilinmektedir[3]. Fakat bu avantaj kalkış ve iniş gibi düşük hızlarda yapılması gereken uçuşlarda dezavantaja dönüşebilir. ZANKA-II insansız hava aracının kanat ucu tasarımını her uçuş safhasında daha verimli kılabilmek için menteşeli bir mekanizma yardımı ile aerodinamik verimliliğin artılması hedeflenmiştir. Morphinglet tasarımı kanat ucuna yerleştirilen servo sistem yardımı ile hareket etmektedir. Uçuş kontrol sisteminde alınan sinyaller yardımı ile harekete geçen servo sisteme bağlı olan kanat ucu yapısı hareket ederek hava aracını bütün uçuş safhalarında daha verimli olmasını sağlamaktadır. 
Şekil 3. Zanka II insansız hava aracı

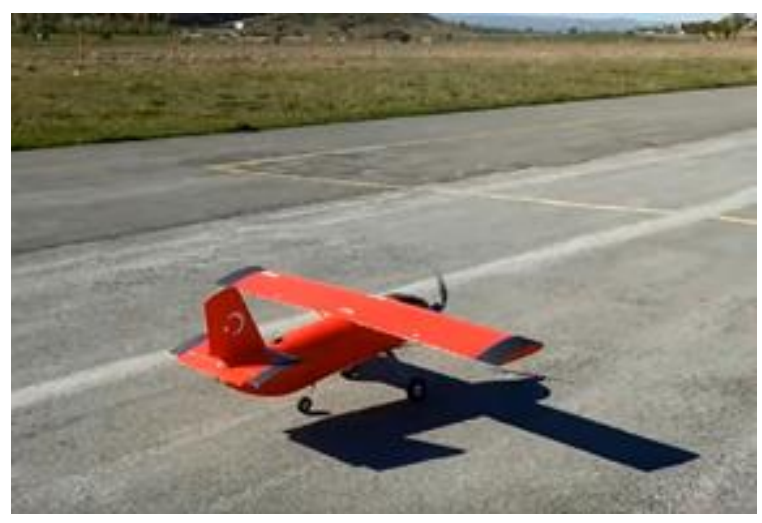

Hava aracının havada tutunması ağırlık kuvvetinin tersi yönde bir kuvvet gerekmektedir. Taşıma kuvvetine etki eden parametreler şunlardır; kanatın şekli, kanat alanı, havanın yoğunluğu ve havanın hızıdır. Statik kanatlarda bu parametrelerden sadece havanın hızı değişkenlik gösterirken başkalaşan kanat ucu tasarımına sahip hava araçları kanat alanında değişimine izin vermektedir. Hava aracının havaya tutunabilmesi için gereken hıza stall hızı denir. Stall hızında iken hava aracının kanatlarının ürettiği taşıma kuvveti hava aracının ağırlığına eşittir. Stall hızını düşürmek için birçok yöntem bulunmaktadır. Başkalaşan kanat ucu tasarımı stall hızını düşüren yöntemlerden birisidir.

Tasarım yaparken dikkat edilmesi gereken diğer önemli parametre ise sürükleme kuvvetidir. Uçuş yönünün tersine yönde gerçekleşen bu kuvvet hava aracına fren etkisi oluşturmaktadır. Bu yüzden sürükleme kuvvetinin az olması istenir. Sürükleme kuvveti hava aracının hızının karesi ile doğru orantılıdır. Sürükleme kuvvetlerinden vorteks sürüklemenin azaltılması için kıvrık kanat ucu tasarımı kullanılmıştır. Düşük hızlarda vorteks sürüklemesi de az olacağından dolayı düşük hızlarda kıvrık kanat ucu hava aracımızın performansına avantajdan çok dezavantaj sunmaktadır. Bundan dolayı başkalaşan kanat ucu tasarımına ihtiyaç duyulmuştur.

Hava araçlarında daha yüksek taşıma kuvveti üretmek için bazı yedek kontrol yüzeyleri bulunmaktadır. Bu yardımcı kontrol yüzeyleri genellikle hava aracının hızının nispeten düşük olduğu kalkış ve iniş gibi uçuş safhalarında kullanılmaktadır. Kaldırma kuvvetini artırmak için kanat alanının artırılması ve kanat şeklinin değiştirme işlemleri yapılmaktadır. Bu işlem için genellikle kanat hücum ve firar kenarına hareketli parça eklenmesi yapılırken başkalaşan kanat ucu tasarımında kanat ucuna hareketli parça eklenmiştir.

Sabit kanatlı hava aracının kanatlarındaki kaldırma kuvveti aşağıdaki formül ile hesaplanmaktadır;

$$
L=C_{l} \times \frac{1}{2} \times \rho \times V^{2} \times A
$$

\section{$\mathrm{L}=$ Kaldırma kuvveti}

$\mathrm{Cl}=$ kaldırma kuvveti katsayısı (airfoil yapının şekline ve hücum açısına bağlıdır)

$\rho=$ hava yoğunluğu

$\mathrm{v}=$ hava hiz1

$\mathrm{A}=$ kanat alanı

Bu çalışmada insansız hava araçlarına başkalaşan kanat ucu tasarımı eklenerek bilgisayar ortamında hesaplamalı akış dinamiği yöntemi ile simülasyonu yapılmaya çalışılmıştır. Bu yöntem ile akışkan hava moleküllerinin hava aracı üzerine etkilerini algoritmalar ile bilgisayar ortamında analiz edilmesidir. Günümüz hava araçlarının aerodinamik olarak daha mükemmel yapıda ve verimli olmasında önemli bir role sahip olan bu yöntem hava aracı üretilmeden önce sürükleme kuvvetinin etkilerini görmemize yardımcı olmuştur.

\section{Tartışma}

Her iki kanatta yer alan başkalaşan kanat ucu tasarımı simetrik bir şekilde aynı anda hareket ederek emniyetli ve stabil bir hareket için çok önemlidir. Kanat içerisine yer alan servo mekanizması yardımı ile sistem çalışmaktadır. Servo mekanizmasına bağlı mekanik bağlantı yardımı ile her iki kanat ucu yapısının hareket etmesi planlanmaktadır. Bu sistemi oluşturan komponentler her bir kanat için kanat ucuna hareket kabiliyeti vermesi için menteşe, menteşeyi hareket ettirebilmesi için mekanik bağlantılar, mekanik bağlantıyı hareket ettirmesi için servo motor, servo motora elektrik sinyallerini veren uçuş kontrol bağlantılarını sağlaması için elektrik kablolarından oluşmaktadır.

Başkalaşan kanat ucu hareket ettikten sonra kanat alanı dikey duran winglet alanı kadar uzamaktadır. Kanat alanının uzaması ile kaldırma kuvveti artacak ve stall hızı düşecektir. Stall hızı düşen hava aracı daha kısa mesafeden havalanma kabiliyetine sahip olacaktır. Kıvrık kanat ucu yapısı bozulduğundan vorteks sürüklemesi artacaktır fakat düşük hava hızında etkisi az olacaktır. 


\section{Kaynakça}

1. Biometric spiroid winglets for lift and drrag control. Joel E. Guerrero, Dario Maestro, Alessandro Bottaro. 67-80, s.1.: C.R.Mecanique, 2012, Cilt 340.

2. Graham W., Winglets - All Shapes, Sizes - and Uses. aviationweek.com. https://aviationweek.com/blog/winglets-all-shapes-sizesand-uses

3. Aerodynamic design optimization studies of a blended-wing-body aircraft. Lyu, Z., Martins, Joaquim R. A.,. 5, s.1. : journal of aircraft, Cilt 51.

4. Pinkstone, Adam. The devolepment of a mechanism for the actuation of a morphing winglet. behance.net. [Çevrimiçi] [Alıntı Tarihi: 1504 2019.] https://www.behance.net/gallery/21233351/Boeing-737-Morphing-Winglet.

5. Uzun, Metin. İnsansız hava araçlarının aerodinamik performansının geometrik değişiklikler ile iyileştirilmesi. kayseri : s.n., 2018.

6. Prakash, Paudel. Aerodynamic aspect in the devolepment of morphing winglet for a regional aircraft . Toronto, Canada : s.n., 2015.

7. Preliminary design of an actuation system for a morphing winglet. I. Dimino, B.D. Giampaolo. s.l. : 8th International Conference on Mechanical and Aerospace Engineering, 2017.

8. Aero-structural design optimization of a morphing wingtip. L. Falcao, A. Suleman, A. A. Gomes. s.l. : journal of intelligent material system and structures, 2011, Cilt 22.

9. ÇELİK H. , OKTAY T., TÜRKMEN I.( 2016) Model Predictive Control and Robustness Test of The Unmanned Aerial Vehicle (Zanka-I) in Various Turbulence, Journal of Aeronautics and Space Technologies, cilt.9, ss.31-42, 